\title{
Characteristic Cerebrovascular Findings Associated with ACTA2 Gene Mutations
}

\author{
Andrew Zhang, Alexandria Jo, Karen Grajewski, John Kim
}

Keywords: Vascular neurology, Genetics - clinical

doi:10.1017/cjn.2019.20

Can J Neurol Sci. 2019; 46: 342-343

A specific mutation (Arg179) of the ACTA2 gene has previously been described to cause a syndrome of multisystemic smooth muscle dysfunction with an extremely characteristic cerebrovascular appearance. ${ }^{1}$ Accurate neuroimaging diagnosis of this entity is important as this syndrome predisposes to complications such as early-onset ischemic stroke and ascending thoracic aortic aneurysm. ${ }^{2,3}$ The following case demonstrates a previously undescribed ACTA2 mutation (Met46) with an identical cerebrovascular imaging appearance to that of $\operatorname{Arg} 179$ mutations, but a less severe overall phenotype.

A 19-year-old male with history of patent ductus arteriosus requiring ligation presented for a routine health maintenance exam, where abdominal and femoral bruits were heard. An echocardiogram then demonstrated a mildly dilated aortic root measuring 3.7-4.0 cm, and MRA of the body demonstrated left common iliac artery stenosis. MRA of the head and neck demonstrated dilation of the petrous and cavernous portions of the internal carotid arteries bilaterally, with abrupt tapering of the clinoid and supraclinoid segments, and an abnormally straightened appearance of the posterior, middle, and anterior cerebral arteries (Figure 1). A follow-up MRI performed a year later demonstrated a few scattered foci of T2 prolongation in the white matter, an imaging feature often seen in patients with ACTA2 gene mutations from small vessel ischemic injuries (Figure 2).

An aortopathy gene panel test was performed to evaluate the following genes: ACTA2, CBS, COL3A1, COL5A1, COL5A2, FBN1, FBN2, FLNA, MAT2A, MEDI2, MFAP5, MYH1, MYLK, NOTCH1, PRKG1, SK1, SLC2A10, SMAD3, SMAD4, TGFB2, TGFB3, TGFBR1, and TGFBR2. Results confirmed a heterozygous Met46Arg mutation in the ACTA2 gene, which at

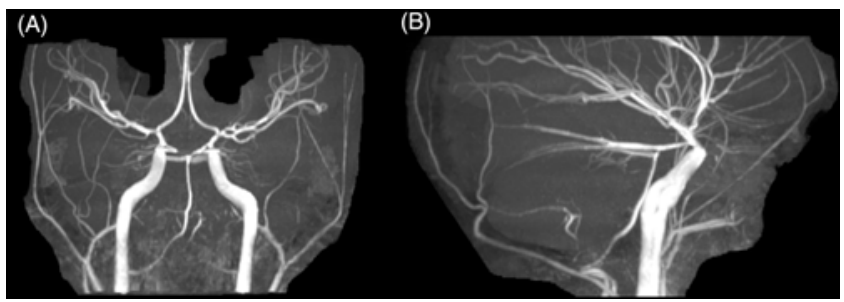

Figure 1: Coronal (A) and sagittal (B) maximum intensity projection imaging from head-and-neck MRA demonstrates dilation of the extradural carotid arteries bilaterally, with abrupt tapering of the intraclinoid carotid artery, and straightened posterior, middle, and anterior cerebral arteries. the time was characterized as a "variant of uncertain significance." Of note, this genetic variant has now been classified as a likely pathogenic variant based on review of data in context of the 2015 ACMG Standards and Guidelines for the Interpretation of Sequences Variants. ${ }^{4}$ Further clinical evaluation found no additional signs or symptoms of multisystemic smooth muscle dysfunction syndrome, such as congenital mydriasis, hypotonic bladder, or pulmonary hypertension, as has been described previously with other ACTA2 mutations. ${ }^{1-3}$ The parents tested negative for the Met46Arg mutation in the ACTA2 gene, indicating that this mutation likely arose de novo.

ACTA2 mutations can have characteristic cerebrovascular findings, allowing the neuroradiologist to suggest a specific genetic diagnosis based on imaging findings alone. These cerebrovascular findings have previously been specifically attributed to heterozygous Arg179 ACTA2 mutations, and include ectasia and stenosis of the internal carotid artery, a straightened course of the cerebral arteries, and an absence of basal collateral vessels. ${ }^{1}$ However, unlike our case, Arg179 ACTA2 mutations cause a more diffuse syndrome of multisystemic smooth muscle dysfunction. ${ }^{3}$ This novel case demonstrates a previously undescribed ACTA2 mutation with cerebrovascular features identical to Arg179 ACTA2 mutations, but a less severe overall phenotype.

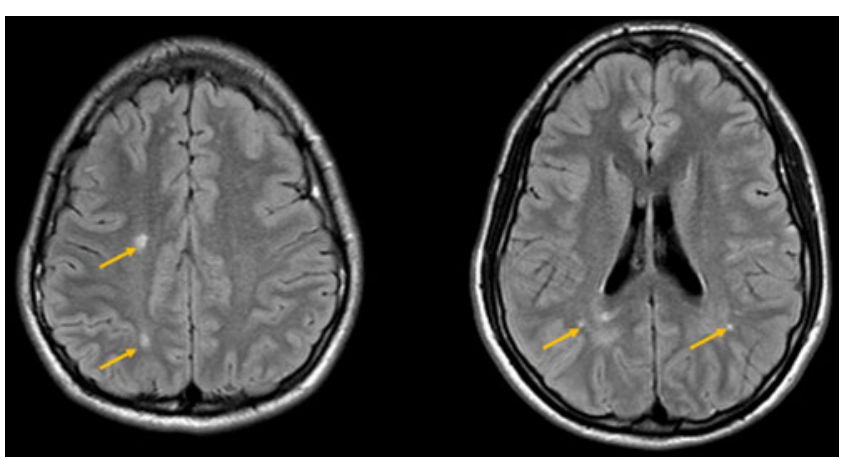

Figure 2: Follow-up MRI brain, performed a year later, demonstrates a few scattered foci of $T 2$ prolongation in the white matter (yellow arrows).

From the Department of Radiology, University of Michigan, Ann Arbor, Michigan, USA

Received July 30, 2018. Final Revisions Submitted December 17, 2018. Date of AcCePtance February 18, 2019.

Correspondence to: Andrew Zhang, Department of Radiology, University of Michigan, 1500 East Medical Center Drive TC2910L, Ann Arbor, Michigan 48109-0326, USA. Email: andzhang@med.umich.edu 


\section{DisClOSURES}

The authors have no conflicts of interest to declare.

\section{Statement of Authorship}

All authors contributed equally to the manuscript.

\section{REFERENCES}

1. Munot P, Saunders DE, Milewicz DM, et al. A novel distinctive cerebrovascular phenotype is associated with heterozygous Arg179 ACTA2 mutations. Brain. 2012;135(Pt 8):2506-14.
2. Guo DC, Papke CL, Tran-Fadulu V, et al. Mutations in smooth muscle alpha-actin (ACTA2) cause coronary artery disease, stroke, and moyamoya disease, along with thoracic aortic disease. Am J Hum Genet. 2009;84:617-27.

3. Milewicz DM, Ostergaard JR, Ala-Kokko LM, et al. De novo ACTA2 mutation causes a novel syndrome of multisystemic smooth muscle dysfunction. Am J Med Genet A. 2010; 152A:2437-43.

4. Richards S, Aziz N, Bale S, et al. Standards and guidelines for the interpretation of sequence variants: a joint consensus recommendation of the American College of Medical Genetics and genomics and the association for molecular pathology. Genet Med. 2015;5:405-24. 